\title{
Dynamics of Growth of Silica Particles from Ammonia-Catalyzed Hydrolysis of Tetra-ethyl-orthosilicate
}

\author{
T. MATSOUKAS AND ERDOGAN GULARI ${ }^{1}$ \\ Department of Chemical Engineering, University of Michigan, Ann Arbor, Michigan 48109
}

Received May 6, 1987; accepted August 12, 1987

\begin{abstract}
The $\mathrm{NH}_{3}$-catalyzed formation of colloidal silica particles from tetra-ethyl-orthosilicate (TEOS) in methanol and ethanol is studied by means of light scattering and Raman spectroscopy. We find that the growth is characterized by an incubation period after which no significant nucleation takes place. The particles have uniform, non-fractal structure and show low polydispersity. In the presence of excess water, the rate-limiting step is the hydrolysis, which is a first-order process in the orthosilicate concentration. (C) 1988 Academic Press, Inc.
\end{abstract}

\section{INTRODUCTION}

Base- and acid-catalyzed hydrolysis and condensation of silicon alkoxides has become of interest because of the potential use in glass and ceramic processing. Alkoxides, which can also be viewed as esters of the weak silicic acid $\mathrm{Si}(\mathrm{OH})_{4}$, undergo hydrolysis in the presence of water and subsequently form a siloxane network by a condensation reaction which is often termed a "polymerization." This reaction takes place at neutral $\mathrm{pH}$ but it is accelerated in both acidic and basic environment. Generally speaking, an acidic environment promotes the formation of a gel while a basic environment favors the formation of a stable sol (1).

Spectroscopic techniques have been widely used to study the kinetics of this reaction system. IR and Raman spectroscopy provide useful information about the kinetics of hydrolysis through the vibrational modes of the orthosilicate (2-4). They are rather insensitive though to the products of hydrolysis and although some band assignments have been offered (4), a quantitative description is not easy. NMR has been used to obtain information on a molecular level and many intermediate spe-

\footnotetext{
${ }^{1}$ To whom correspondence should be addressed.
}

cies have been identified as precursors of the silica network (5-8) but little information has been obtained on the extent of growth, since large species give rise to broad and weak resonances (6). Scattering techniques are more suitable for studying the growth of the polymeric units and SAXS has been successfully used to study their size and structure $(5,9-$ 11). In their majority, however, these works have focused on the conditions that produce a gel, namely high orthosilicate concentration, low water-to-orthosilicate ratio, and acidic or weakly basic environment.

The presence of ammonia promotes the formation of spherical particles, whether the final result is a gel $(11,12)$ or a stable suspension $(1,13)$. The production of uniform-size colloidal silica from alkoxides was first reported by Stoeber et al. (13), who used various alkoxides in pure alcohols and alcohol mixtures and investigated the shape and distribution spread of the particles as a function of the water and ammonia content. An extensive characterization study of the sol by means of light scattering, electron microscopy, and ultracentrifugation was reported by Van Helden et al. (14). These studies have established the picture of spherical particles of low polydispersity and sizes which range from a few 
nanometers to almost a micron as the ammonia concentration is increased. Keefer (9) used SAXS to monitor the formation of small particles by hydrolyzing TEOS in a weakly basic ethanol solution. He observed that the growth of the particles was independent of the size of the particle, and concluded that the growth was limited by the slow hydrolysis. It must be noted, however, that he worked at low water/TEOS ratios which are expected to inhibit the hydrolysis step.

The formation of a stable sol of spherical and relatively monodisperse silica particles can be qualitatively understood in terms of surface stabilization and the competing effect of nucleation and growth. At high $\mathrm{pH}$, the particle surface attains a negative charge and this stabilizes the sol against aggregation. The low spread of the distribution is indicative of a process in which nucleation is limited to the early stages and the "monomer" reacts preferentially with existing particles rather than nucleating. The spherical shape of the particles can be accounted for by simple growth models based on monomer addition to the surface of an existing particle in the presence (15) or absence (9) of a screened coulombic potential. This picture though is qualitative and does not address the fact that increasing $\mathrm{NH}_{3}$ promotes the formation of larger particles. The picture becomes even more complicated when we consider that the active monomer (hydrolyzed orthosilicate) is continuously released by the hydrolysis reaction which proceeds at a time scale comparable to that of the growth.

The purpose of this work has been to get information about both the dynamics of nucleation and growth and the kinetics of hydrolysis in the presence of ammonia. Information about the dynamics was obtained by the scattered intensity and the hydrodynamic radius as measured by laser light scattering. The kinetics were followed by Raman scattering. This last method also allowed us to obtain simultaneously information about the rate of growth by making use of Rayleigh scattering of the plasma lines.

\section{LIGHT SCATTERING FROM A SUSPENSION} OF PARTICLES

The intensity of light scattered at an angle $\theta$ by a suspension of particles is given as (16)

$$
I=\sum i^{2} n_{i} P\left(q r_{i}\right)
$$

where $n_{i}$ is the number concentration of particles of mass $i$ (in units of monomer), $r_{i}$ the radius of the particle, $q=(4 \pi n / \lambda) \sin (\theta / 2)$ the scattering wavevector, and $P\left(q r_{i}\right)$ the form factor, which is determined by the particle geometry. The above equation is valid for dilute systems, i.e., systems for which $2 \pi / q \gg L$, where $L$ is the mean interparticle distance, roughly equal to $N^{1 / 3}, N$ being the total number density of the particles (17). In the Guinier region $(q r<1)$ the form factor is given by (9)

$$
P(q r)=e^{-(q r)^{2} / 5} \text {. }
$$

Assuming that the particle distribution has relatively narrow spread, the intensity takes the form

$I=\left(\sum i^{2} n_{i}\right) P(q r)=M_{2} P(q r)=\frac{M_{1}^{2}}{M_{0}} P(q r)$,

where $r$ is an average radius and $M_{k}=\sum_{i} i^{k} n_{i}$ is the $k$ th-order moment of the distribution. It can be easily verified that $M_{0}$ is the total number of particles and $M_{1}$ their mass. Note that the above summations refer to the particle population, without including the monomer. The justification for this comes from the experimental observation that the intensity of a solution of unhydrolyzed monomer is within the solvent-noise background which implies that the monomer contribution to the intensity is negligible. This is even more so when sizable particles have been formed whose contribution to the intensity is dominant due to the masssquared dependence.

When the number of particles $M_{0}$ remains constant, i.e., the monomer is consumed in reaction with existing particles (growth) rather than with unreacted monomer (nucleation), 
the intensity provides a direct measure of the total particle mass and $M_{1}$ can be written as

$$
M_{1} \sim\left(\frac{I}{P(q r)}\right)^{1 / 2} .
$$

In such a case, the extent of the reaction and product formation can be readily followed by intensity measurements. The assumption of constant $M_{0}$ which is necessary to provide this simple relation is justified by the experimental observation $(13,14)$ that the resulting particles are of uniform size and shape, which is consistent with a process where nucleation is limited to the early stages and once the nuclei have been formed, growth is the dominant reaction. On the experimental side, direct verification of this assumption can come from Eq. [3]. In the general case of particles of fractal dimension $D_{f}$, the total mass scales with the radius as $M_{1} \sim M_{0} r^{D_{f}}(18)$ and the 2 nd moment takes the form

$$
M_{2}=\frac{I}{P(q r)} \sim M_{0} r^{2 D_{f}} .
$$

For constant $M_{0}$, a $\log$ - $\log$ plot of $M_{2}$ vs $r$ should yield a straight line with a slope of $2 D_{f}$. For uniform (non-fractal) particles, $2 D_{f}=6$.

\section{EXPERIMENTAL}

The experiments were done with dilute solutions of TEOS in ethanol and methanol, typically 0.087 mole/liter. Concentrated ammonium hydroxide $\left(30 \%\right.$ in $\mathrm{NH}_{3}$ ) was diluted in deionized water to produce solutions of desired normalities. Samples for the light scattering experiments were filtered through a 0.2$\mu \mathrm{m}$ microfilter and centrifuged.

In order to minimize interparticle interactions and multiple scattering during growth experiments, light scattering has to be performed in dilute solutions. For the experiments shown here, the composition of the solution was $0.0087 \mathrm{~mole} / \mathrm{liter}$ TEOS, 1.2-1.6 moles/liter $\mathrm{NH}_{3}$, and 3.2 moles/liter water.

Raman experiments must be conducted at higher TEOS concentrations because of de- tectability limitations. Also a lower ammonia concentration is necessary since large particles obscure the spectrum through intense Rayleigh scattering. Typical concentrations were $0.4 \mathrm{~mole} / \mathrm{liter}$ TEOS, $0.65 \mathrm{~mole} / \mathrm{liter} \mathrm{NH}_{3}$, and 8.5 moles/liter water. In all the experiments, the water-to-orthosilicate ratio was well above the stoichiometric $4 / 1$ for complete hydrolysis and $2 / 1$ for the overall reaction (for complete condensation to $\mathrm{SiO}_{2}$ ).

Light scattering experiments were performed using an argon ion laser operating at the 514.5-nm line. Raman spectra were obtained at an excitation wavelength of $488 \mathrm{~nm}$.

Particle sizes were measured simultaneously with the intensity using dynamic light scattering. The radius was calculated from the Stokes-Einstein equation

$$
r=\frac{k T}{6 \pi \eta D_{z}}
$$

where the $z$-average diffusion coefficient $D_{z}$ was obtained from the initial slope of the autocorrelation function by the use of a secondorder cumulant expansion.

All the experiments reported here were done at $25^{\circ} \mathrm{C}$.

\section{RESULTS}

\section{(a) Light Scattering}

Because of the low miscibility between the unhydrolyzed orthosilicate and water, a mutual solvent must be used, such as an alcohol (methanol and ethanol in this study). The solvent used and the concentration of ammonia are two factors upon which the final size of the particles strongly depends. This is shown in Fig. 1 for a TEOS concentration of 0.087 mole/liter. In both solvents increasing concentration of ammonia results in larger particles. This effect is stronger in methanol. Particles grown in ethanol attain larger sizes than those in methanol and this is more pronounced in the low ammonia concentration region. This difference becomes progressively smaller as the concentration of ammonia in- 


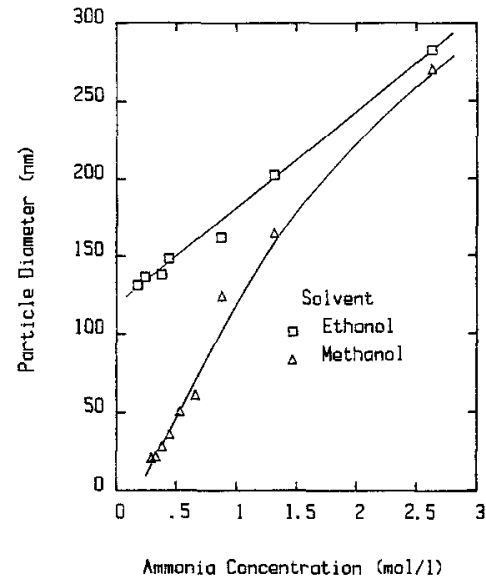

FIG. 1. The effect of ammonia concentration on the particle size. Concentration of TEOS $=0.087$ mole/liter.

creases and, eventually, particles in either solvent grow to comparable sizes.

The same factors that determine the size of the particles also affect directly the rate of growth. Figure 2 shows the scattered intensity from particles grown in the two alcohols, and under different concentrations of ammonia. In all cases we initially observe an induction period during which the intensity remains constant at the background level. Dynamic light scattering produces no meaningful autocorrelation functions and no particle sizes can be determined during this period. This is followed by a steady increase in intensity which finally reaches a steady-state value when all the monomer has been consumed. The evolution of the particle diameter is even faster, and interestingly enough, the smallest sizes detected are already within $50-70 \%$ of their final values. The near exponential decay of the autocorrelation function suggests narrow particle distributions. Polydispersities, customarily reported as $K_{2} / K_{1}^{2}$, where $K_{i}$ is the $i$ th-order cumulant, were typically found to be less than 0.05 . Upon increasing the concentration of ammonia we observe a shorter incubation time and overall faster growth rate. The final intensity is also higher, reflecting the production of larger particles, but this is not

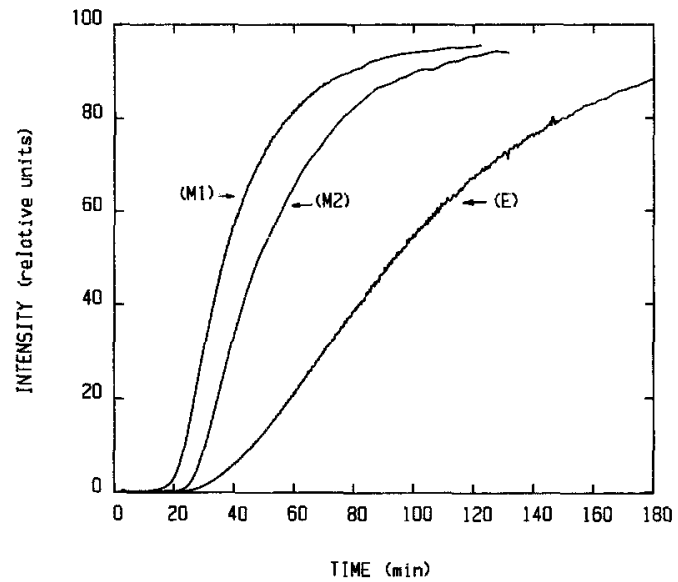

FIG. 2. The scattered intensity at $\theta=120^{\circ}$ from particles grown in methanol (M1, M2) and ethanol (E). The concentration of ammonia is 1.6 moles/liter for $E$ and $\mathrm{Ml}$ and 1.2 moles/liter for $\mathrm{M} 2$.

shown in Fig. 2 because intensities have been normalized to their steady-state value. When the growth takes place in ethanol, the overall picture is the same, except that the growth rate is considerably slower. While not shown here, increasing the concentration of ammonia results in faster growth, as with methanol.

The log-log plot of $M_{2}$ vs $r$ is shown in Fig.

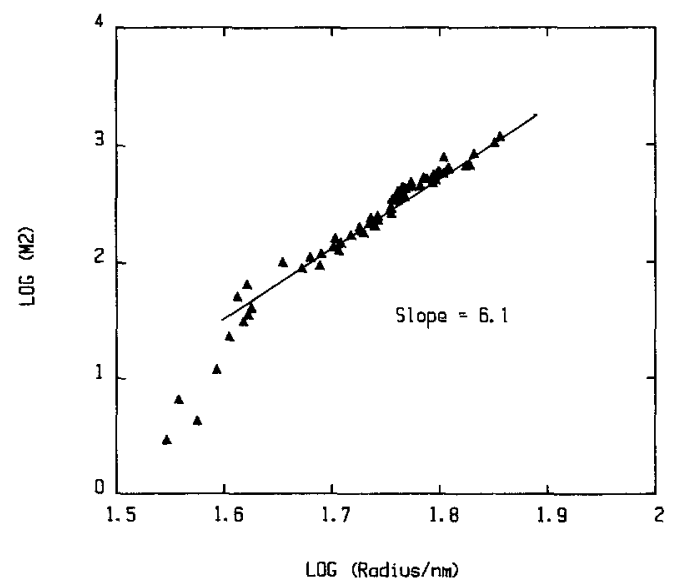

FIG. 3. The second moment vs the radius for sample (E). The second moment $M_{2}$ is calculated as $I / P(q r)$. The linearity of this plot shows that the number of particles is conserved. 
3 for the particles grown in ethanol. We see a linear dependence and a least-squares fit produces a slope of 6.1. From this we conclude that the number of particles remains constant, at least after the particles have attained $50 \%$ of their final size (corresponding to $13 \%$ conversion of the monomer). The larger slope in the small size region of the graph could be attributed to ongoing nucleation, but it must be noted that sizes in this region correspond to weak autocorrelations due to the low scattered intensity. Second, from the slope of this graph we obtain $D_{f}=3.1 \pm 0.05$, which indicates uniform, non-fractal particles. While this cannot be considered an accurate determination of the fractal dimension, it is in agreement with the results by Keefer (9) for conditions of complete hydrolysis.

The high power of the intensity-radius correlation is responsible for the fact that we do not detect particle sizes during nucleation: the intensity which is scattered by particles with a diameter $35 \%$ of the final diameter is only about $0.2 \%$ of the final scattered intensity. This intensity is most often within the background level and is insufficient to produce any accurate autocorrelation functions within a reasonable amount of collection time.

The intensity was analyzed assuming simple first-order kinetics. The total mass $M_{1}$ calculated from Eq. [4] and plotted as $\ln \left(1-M_{1} /\right.$ $\left.M_{1}(\infty)\right)$ vs time is shown in Fig. 4. Except for the incubation period, this simple form describes remarkably well the growth in both ethanol and methanol. This indicates that the equation of growth is of the form

$$
\frac{d M_{1}}{d t}=k\left(c-M_{1}\right)
$$

Since $c-M_{1}$ can be identified as the unreacted monomer, it appears that the growth depends upon the availability of the monomer, but not explicitly upon the size of the particle. If the number of reactive sites depends on the size of the particle, the equation of growth should be of the form

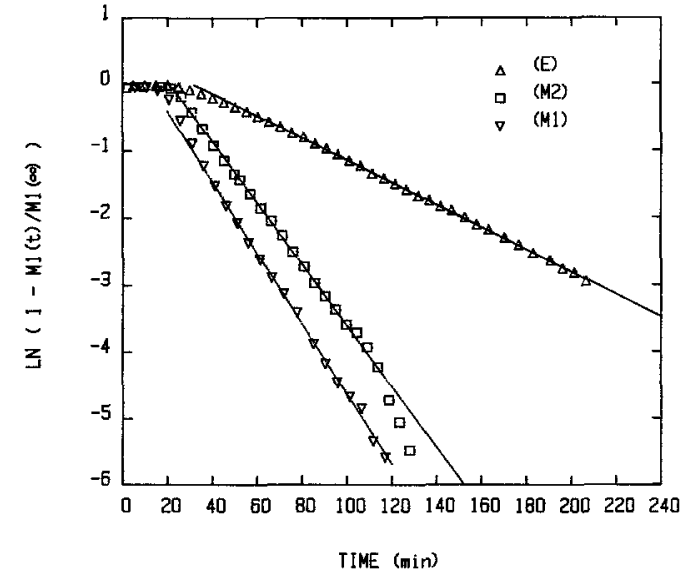

FIG. 4. Linearized plot of the particle mass $M_{1}$ vs time. $M_{1}$ is calculated as $(I / P(g r))^{1 / 2}$. The linearity shows that the growth is first order in the concentration of the unreacted monomer.

$$
\frac{d M_{1}}{d t}=k^{\prime}\left(c-M_{1}\right) M_{1}^{a}
$$

where for surface reaction $a=\frac{2}{3}$. The fact that explicit dependence on $M_{1}$ is absent leads to the conclusion that the growth is limited by the rate of monomer release and this is first order in the orthosilicate.

Table I summarizes the results from the growth experiments and shows the rate constants extracted from the slope of the linear plots of Fig. 4. We see that under otherwise identical conditions the growth rate in ethanol is one-third of that in methanol.

\section{(b) Raman Spectra}

Raman spectroscopy was used in order to obtain information simultaneously about both the kinetics of hydrolysis and the extent of the polymerization reaction. The hydrolyzing monomer can be readily followed through its characteristic band at $664 \mathrm{~cm}^{-1}$ due to the Si$\mathrm{O}-\mathrm{Et}$ bond. The same is not true for the $\mathrm{Si}-$ O-Si network, which lacks any characteristic bands that can lead to a quantitative description of the growth. To overcome this problem, we made use of plasma lines. These are sharp 
TABLE I

\begin{tabular}{lcccc}
\hline Sample & $\begin{array}{c}\text { TEOS } \\
\text { (mole/liter) }\end{array}$ & $\begin{array}{c}\text { Ammonia } \\
\text { (moles/liter) }\end{array}$ & $\begin{array}{c}\text { Diameter } \\
(\mathrm{nm})\end{array}$ & $\begin{array}{c}k \\
\left(\mathrm{~min}^{-1}\right)\end{array}$ \\
\hline $\mathrm{M} 1$ & 0.0087 & 1.6 & 57 & 0.052 \\
$\mathrm{M} 2$ & 0.0087 & 1.2 & 46 & 0.046 \\
$\mathrm{E}$ & 0.0087 & 1.6 & 160 & 0.017 \\
\hline
\end{tabular}

lines normally found in the beam and under conventional operation a grating is used to remove them. However, if they are left in the beam, they will appear in the spectrum only through Rayleigh scattering in the presence of suitable scatterers. It is important to note that these lines convey no chemical information and their position in the spectrum is fixed, independent of the chemical nature of the species that scatter them. Their intensity depends on the size and concentration of the scatterers, according to the principles outlined in the previous sections. Monitoring their intensity as a function of time provides a very simple way to follow the particle growth, along with the evolution of the Raman bands of the orthosilicate. This allows a direct comparison of the hydrolysis rate to that of the growth.

Figure 5 shows typical spectra of TEOS in

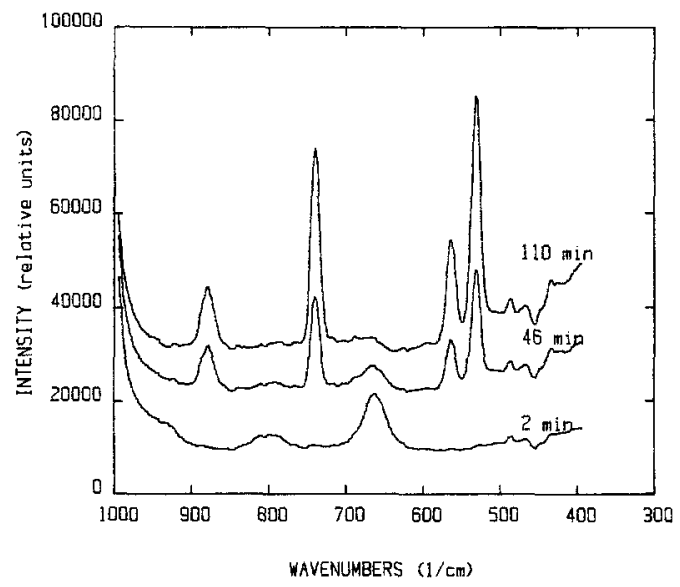

FIG. 5. Raman spectra of TEOS in methanol at different times. The peaks at 664 and $807 \mathrm{~cm}^{-1}$ are due to the orthosilicate. The rising band at $880 \mathrm{~cm}^{-1}$ is due to the ethanol released during the hydrolysis. The growing lines at 740,563 , and $531 \mathrm{~cm}^{-1}$ are plasma lines. methanol at different times. The peaks at 807 and $664 \mathrm{~cm}^{-1}$ are due to the orthosilicate and they diminish with time. The emerging band at 880 is due to ethanol produced during the hydrolysis. The sharp lines at 740,563 , and 531 wavenumbers are all plasma lines and they increase with time. Upon the completion of the reaction, the spectrum consists of the bands of methanol and ethanol and the plasma lines. No new bands characteristic of the silica network were detected in the spectral region that we monitored. Figure 6 shows the evolution of the integrated intensity of the orthosilicate $\left(664 \mathrm{~cm}^{-1}\right)$, ethanol $\left(880 \mathrm{~cm}^{-1}\right)$, and a plasma line $\left(740 \mathrm{~cm}^{-1}\right)$. The evolution of the plasma line exhibits the characteristic incubation period seen in the light scattering experiments, but no such a feature is observed in TEOS or ethanol.

Following the results of the light scattering experiments, the integrated intensity of these bands was analyzed by assuming first-order kinetics. The linearized forms are plotted as $\ln (y)$ vs time in Fig. 7, where $y$ is defined as

$$
\begin{aligned}
y_{\text {TEOS }} & =I(t) / I(0) \\
y_{\text {EtOH }} & =1-I(t) / I(\infty) \\
y_{\text {plasma }} & =1-\sqrt{I(t) / I(\infty)} .
\end{aligned}
$$

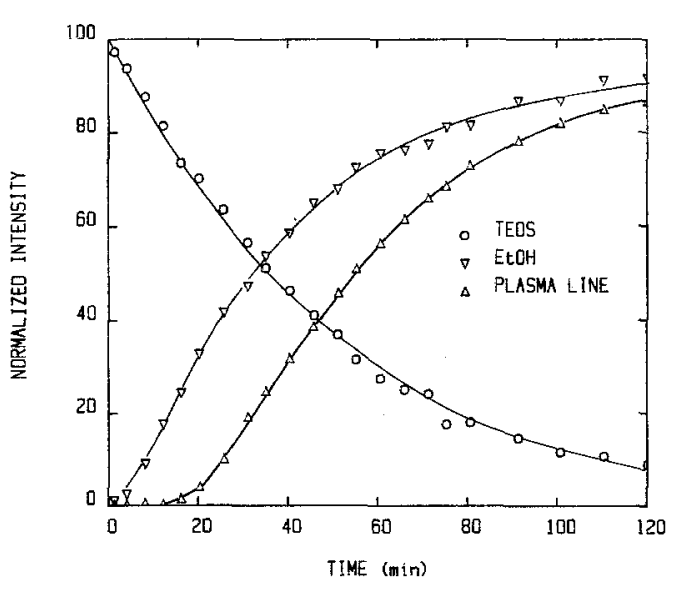

FIG. 6. Integrated intensity of the Raman bands as a function of time. Notice the incubation time in the evolution of the plasma line. This feature is not observed with the bands of TEOS and ethanol. 


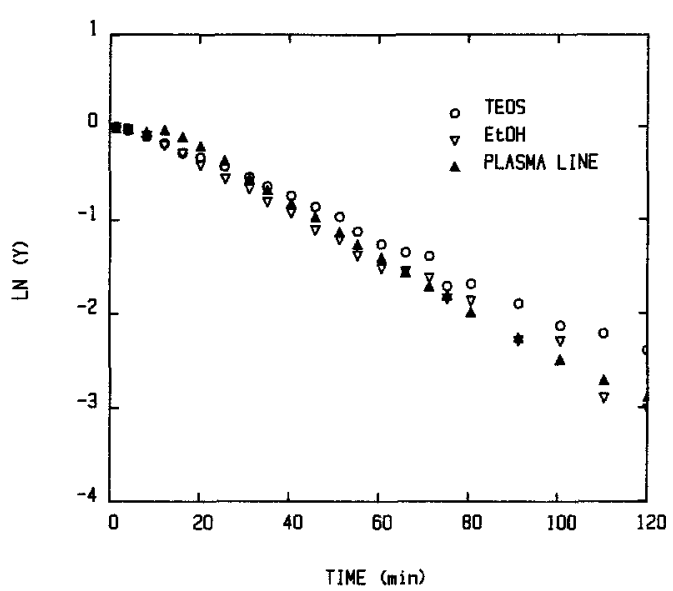

FIG. 7. Linearized plot of the intensity of the Raman and plasma bands (see the text for the definition of $y$ ). All the points fall on a single line.

It can be seen that all the points seem to fall on a single line with slope equal to the pseudofirst-order rate constant of hydrolysis. The orthosilicate appears to evolve with a somewhat smaller slope but the production of ethanol correlates very well with the growth of the plasma line. A similar plot obtained from particles grown in ethanol is shown in Fig. 8. We observe much slower kinetics, partly because lower concentration of ammonia was used to avoid the formation of very large particles. The correlation between the TEOS band and the plasma line is good. The deviations observed at longer times are most probably due to the inaccuracies involved in the integration of the weak orthosilicate bands. The ethanol bands are not shown for this sample because the amount released during the hydrolysis is a tiny portion of the excess alcohol present as solvent.

The absence of bands from partly hydrolyzed species is consistent with a fast condensation reaction, although these bands are not expected to be very strong in the dilute samples that we used. These results are in agreement with our light scattering results. The correlation between the rate of growth, as obtained from the plasma line, and the rate of hydrolysis is a direct evidence that the hydrolysis is the rate-limiting step.

\section{DISCUSSION}

It is interesting to observe that the growth process is controlled by the rate of hydrolysis even for large water-to-orthosilicate ratios, but we also realize that this provides a good basis for explaining the experimental observations concerning the base-catalyzed growth in alcohol solutions. These observations can be summarized as follows: (a) Reaction rates are faster in methanol. (b) Under otherwise identical conditions, particles grow larger in ethanol. (c) Ammonia increases the reaction rate and promotes the formation of larger particles. (d) Low water concentration favors the production of larger particles but excess water has the opposite effect $(13,14)$.

The size of the particles is directly related to nucleation. The monomer is consumed in reaction either with monomers to produce nuclei or with existing particles. Factors that promote nucleation must result in smaller particles while factors that favor growth should have the opposite effect. Nucleation can be a kinetic phenomenon resulting from a low monomer-monomer reaction rate. It can also be a dynamic effect determined by the two competing processes, nucleation and growth.

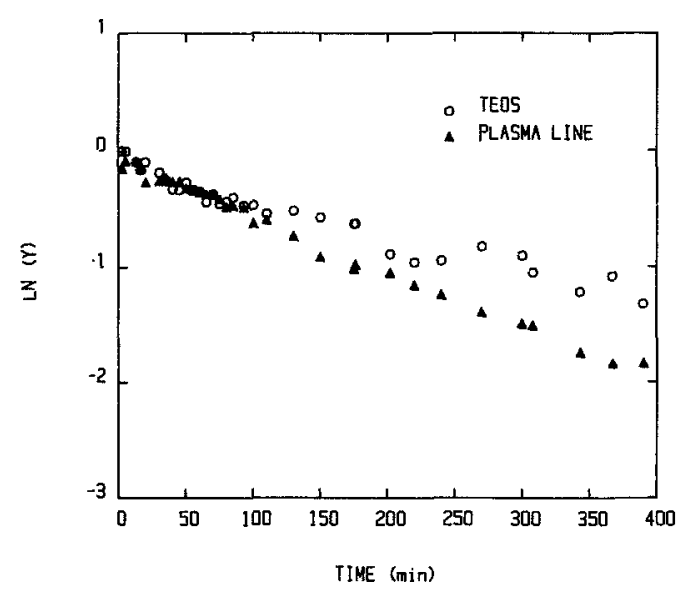

FIG. 8. Linearized plot of the intensity of the Raman and plasma bands from particles grown in ethanol (see the text for the definition of $y$ ). As with methanol, the growth rate follows the rate of hydrolysis. 
In the latter case, hydrolysis plays a dominant role since it controls the availability of the hydrolyzed monomer. If nucleation is defined as the reaction between two monomers, then the nucleation rate is a second-order reaction in the concentration of the active (hydrolyzed) monomer, whereas growth is first order. Monomer-monomer reaction is significant only during the early stages of the process, when the concentration of grown particles (and hence the rate of growth) is low. Under conditions of low concentration of hydrolyzed monomer (hydrolysis the rate-limiting step) and in the presence of already grown particles, the main process of monomer consumption is growth. This is even more so when the stability of the nuclei is taken into account. In this case the apparent order of nucleation is larger than 2 and this introduces an additional bias against nucleation. In the other extreme, when the rate of hydrolysis is faster than the rate of monomer reaction, the increased concentration of hydrolyzed monomer must result in higher nucleation rate and more particles, which also means smaller sizes. Therefore, hydrolysis has a direct effect upon nucleation and the resulting particle size, and if all other factors remain the same, factors that inhibit hydrolysis inhibit nucleation as well and produce larger particles.

The faster kinetics observed under higher ammonia concentrations must be attributed to the higher hydrolysis rate. However, the effect of ammonia goes beyond increasing the rate of monomer release. This is evident by the fact that ammonia also promotes the formation of larger particles. Arguments based on a simple monomer addition model (19) show that under kinetic limitation of monomer release, the rate of growth reflects the rate of release, but the rate of nucleation (and thus the final size) depends on the ratio of the hydrolysis rate to the monomer reaction (polymerization) rate. We think that the effect of ammonia is exactly this, i.e., to promote hydrolysis, but also to promote the polymerization rate to an even higher degree, resulting in faster kinetics (still hydrolysis limited), and larger sizes.

Along the same lines we can predict the behavior under different concentrations of water. In the presence of excess water, the rate of hydrolysis is first order in the orthosilicate and the pseudo-first-order rate constant is a function of the water concentration. We cannot discern much about the reaction order with respect to water, but we expect it to be somewhere between 1 and 4 . In any case, the rate constant must increase with increasing water concentration and this should lead to faster kinetics and smaller particles. Indeed, faster kinetics and smaller particles were observed when instead of pure ethanol we used a 50\% vol solution of water in ethanol. At low (close to stoichiometric) water concentrations we do not expect our arguments to hold. Water is consumed during the hydrolysis and the rate "constant" is not constant any longer.

The effect of the solvent is not easily explained. We speculate that the re-esterification reaction (reverse hydrolysis) is responsible and it can indeed account for our observations. The reversibility of the hydrolysis reaction is known, and it has been experimentally demonstrated by Brinker and co-workers (5), who detected mixed silicate esters in reactions taking place in alcohol mixtures. With higher alcohols, this reaction has been used directly to coat the particle surface with an organophilic layer (14). We may expect then that under excess alcohol the reverse reaction will be significant. Given the fact that methyl orthosilicate is more reactive than its ethyl counterpart, we can understand why the rates are higher in methanol than in ethanol. If in addition to that, the rate of condensation is the same in both alcohols, then larger particles will be produced under conditions of slower hydrolysis, i.e., in ethanol, which is what we experimentally observe. Unfortunately, we failed to detect any Si-O-Me bands in our Raman spectra. This could also be because of the reactivity of the bond and the fact that the dilute samples we used give rise to weak bands. 
Some comments are due with respect to diffusion, especially since first-order processes are often indicative of mass transfer limitations. While the role of diffusion may not be negligible, it cannot account for the observed dynamics. If diffusion were the rate-limiting step, then by decreasing the diffusion coefficient (by increasing the viscosity of medium, for instance) smaller sizes are expected (20), as the slow reaction rate increases the concentration of the hydrolyzed monomer and the nucleation rate. Ethanol has more than twice the viscosity of methanol, yet particles grow much larger in ethanol. In light of the Raman experiments, the evidence points towards hydrolysis as the rate-limiting step. If diffusion is faster than the rate of hydrolysis, then the question is how it compares with the rate of condensation. As long as the overall rate is controlled by the hydrolysis, this question cannot be answered. The relative importance of the two processes will not affect the overall rate of growth. It will affect, though, the internal distribution of mass among the particles and possibly within the particles as well, in terms of fractal or non-fractal structure (21). It seems that at this level the process is controlled by the condensation reaction (9) and this is also supported by the uniform structure of the resulting particles (non-fractal particles), although as shown by Hurd (15), such a result may also be consistent with a diffusion-limited growth in the presence of a screened coulombic particle interaction.

\section{CONCLUSIONS}

In conclusion, we have shown that the ammonia-catalyzed growth of colloidal silica particles from TEOS in a lower alcohol is controlled by the rate of hydrolysis, which under excess water is first order in the orthosilicate. Based on this, most of the experimental observations concerning this process can be understood. We experimentally showed that the number of particles is conserved through the major part of the growth, which implies that nucleation is limited to the early stages of the process. We have offered a quantitative interpretation of our light scattering experiments, and finally, we demonstrated the use of the plasma lines as a means of obtaining simultaneous information on the extent of the condensation reaction along with the chemical information contained in the Raman spectrum.

\section{ACKNOWLEDGMENT}

Financial support of this research by the TTP program of the NSF under Grants CBT830350 and 8701718 is gratefully acknowledged.

\section{REFERENCES}

1. Iler, R., "The Chemistry of Silica," Wiley, New York, 1979.

2. Artaki, I., Brandley, M., Zerda, T. W., and Jonas, J., J. Phys. Chem. 89, 4339 (1985).

3. Prassas, M., and Hench, L. L., in "Ultrastructure Processing of Ceramics, Glasses and Composites," $p$. 100. Wiley, New York, 1984.

4. Klein, L. C., and Kordas, G., in "Better Ceramics through Chemistry, Mater. Res. Soc. Symp. Proc." (C. J. Brinker, D. E. Clark, and D. R. Ulrich, Eds.), Vol. 73, p. 461. Mater. Res. Soc., Pittsburgh, 1986.

5. Brinker, C. J., Keefer, K. D., Schaefer, D. W., Assink, R. A., Kay, B. B., and Ashley, C. S., J. Non-Cryst. Solids 63, 45 (1984).

6. Klemperer, W. G., Maiz, V. V., and Millar, D. M., in "Better Ceramics through Chemistry, Mater. Res. Soc. Symp. Proc." (C. J. Brinker, D. E. Clark, and D. R. Ulrich, Eds.), Vol. 73, p. 15. Mater. Res. Soc., Pittsburgh, 1986.

7. Balfe, G. A., and Martinez, S. L., in "Better Ceramics through Chemistry, Mater. Res. Soc. Symp. Proc." (C. J. Brinker, D. E. Clark, and D. R. Ulrich, Eds.), Vol. 73, p. 27. Mater. Res. Soc., Pittsburgh, 1986.

8. Kay, B. D., and Assink, A., in "Better Ceramics through Chemistry, Mater. Res. Soc. Symp. Proc." (C. J. Brinker, D. E. Clark, and D. R. Ulrich, Eds.), Vol. 73, p. 157. Mater. Res. Soc., Pittsburgh, 1986.

9. Keefer, K. D., in "Better Ceramics through Chemistry, Mater. Res. Soc. Symp. Proc.” (C. J. Brinker, D. E. Clark, and D. R. Ulrich, Eds.), Vol. 73, p. 295. Mater. Res. Soc., Pittsburgh, 1986.

10. Yamane, M., Inoue, S., and Yasumori, A., J. NonCryst. Solids 63, 13 (1984). 
11. Brinker, C. J., Keefer, K. D., Schaefer, D. W., and Assley, C. S., J. Non-Cryst. Solids 48, 47 (1982).

12. Nogami, M., and Moriya, Y., J. Non-Cryst. Solids 37, 191 (1980).

13. Stoeber, W., Fink, A., and Bohn, E., J. Colloid Interface Sci. 26, 62 (1968).

14. Van Helden, A. K., Jansen, J. W., and Vrij, A., $J$. Colloid Interface Sci. 81, 354 (1981).

15. Hurd, A., in "Fractal Aspects of Materials, Extended Abstracts from Symposium N, Fall Meeting of Mater. Res. Soc." (R. B. Laibowitz, B. B. Mandelbrot, and D. E. Passoja, Eds.), p. 51. Mater. Res. Soc., Pittsburgh, 1985.

16. Feder, J., and Jøssang, T., in "Scaling Phenomena in
Disordered Systems" (R. Pynn and A. Skjeltorp, Eds.), p. 99. Plenum, New York, 1985.

17. Pussey, P. N., and Tough, R. J. A., in "Dynamic Light Scattering" (R. Pecora, Ed.), p. 85. Plenum, New York, 1985.

18. Shaefer, D. W., Martin, J. E., Wiltzius, P., and Cannel, D. S., Phys. Rev. Lett. 52, 2371 (1984).

19. Matsoukas, T., and Gulari, E., to be published.

20. Calvert, P., in "Better Ceramics through Chemistry, Mater. Res. Soc. Symp. Proc." (C. J. Brinker, D. E. Clark, and D. R. Ulrich, Eds.), Vol. 73, p. 79. Mater. Res. Soc., Pittsburgh, 1986.

21. Weitz, D. A., Huang, J. S., Ling, M. Y., and Sung, J., Phys. Rev. Lett. 54, 1416 (1985). 